\title{
EFFECTS OF PREHEATED CLUSTERS ON THE COSMIC MICROWAVE BACKGROUND SPECTRUM
}

\author{
KaI-Yang Lin, ${ }^{1,2}$ TaK-Pong Woo, ${ }^{1}$ Yao-Huan Tseng, ${ }^{1,2}$ Lihwai Lin, ${ }^{1}$ And Tzinong Chiueh ${ }^{1,2}$ \\ Received 2002 November 25; accepted 2004 April 26; published 2004 May 11
}

\begin{abstract}
Mounting evidence from X-ray observations reveals that bound objects should have received a significant amount of nongravitational energy in the past. We report an instantaneous heating scheme, for which gases in dense regions receive a temperature increment of several $\mathrm{keV}$ at $z=2$ whereas those in rarified regions remain intact, that can produce bound objects obeying the observed mass-temperature and luminosity-temperature relations. Such heating reduces the Sunyaev-Zel'dovich (SZ) flux by a factor of $3-2$ on a scale of 4'-6'. It exacerbates the need for the matter-fluctuation normalization $\sigma_{8}$ to assume an exceedingly high value $(\sim 1.2)$, if the several-arcminute excess cosmic microwave background anisotropy detected by the Cosmic Background Imager experiment is entirely caused by the SZ effect of intergalactic hot gas. By contrast, we find that the several-arcminute excess detected by Arcminute Cosmology Bolometer Array Receiver can be consistent with the preheated SZ signals of $\sigma_{8}=1$.
\end{abstract}

Subject headings: cosmic microwave background — cosmology: theory — intergalactic medium

On-line material: color figures

\section{INTRODUCTION}

In the hierarchical structure formation model of cold dark matter, gravitationally bound objects obey similarity scaling relations. That is, the mass of a collapsed object scales as the third power of its size and the squared velocity dispersion (or temperature) as the $\frac{2}{3}$ power of the mass. These scaling relations follow from a straightforward dimensional analysis, given that the gravitational collapse occurs much faster than the Hubble expansion and depends on only two parameters-gravitational constant $G$ and Hubble parameter $H$ (Kaiser 1986; Evrard \& Henry 1991; Navarro et al. 1996; Shapiro \& Iliev 2002; Chiueh \& He 2002).

However, X-ray observations in the past years have consistently revealed that the gas component in bound objects violates the self-similar scaling. The observed X-ray luminositytemperature $(L-T)$ relation shows that the cluster temperature appears considerably higher than the scaling $T \propto L^{1 / 2}$ indicates, more so for low-mass clusters than for high-mass clusters (Ponman et al. 1996; Allen \& Fabian 1998; Xue \& Wu 2000). Although the observed mass-temperature $(M-T)$ relation roughly obeys the scaling relation, the temperature is still found higher by $30 \%$ or so than expected from adiabatic structure formation processes (Finoguenov et al. 2001; Allen et al. 2001). Recent $X M M$ observations of metal lines, which directly probe the core temperatures of cooling-flow clusters, further found the efficient cooling not to efficiently reduce the core temperature (Peterson et al. 2003). These evidences all suggest certain nongravitational heat injection to be operative sometimes in the past.

One candidate of heat sources is the outflows from active galactic nuclei (AGNs; Tabor \& Binney 1993; David et al. 2001). Such a mechanism should be more pronounced in the far past, when quasars were highly active, than in the present. Investigators have tested various schemes for heat injection at a very early epoch, $z \geq 4$ (da Silva et al. 2001; Bialek et al. 2001; Borgani et al. 2001a, 2002; Muanwong et al. 2002).

\footnotetext{
${ }^{1}$ Department of Physics, National Taiwan University, Wenshan Chiu, Taipei 116, Taiwan.

${ }^{2}$ Institute of Astronomy and Astrophysics, Academia Sinica, P.O. Box 23141, Taipei 106, Taiwan.
}

Although the observed $L-T$ relation may be recovered, most failed in producing the observed $M-T$ relation. (Nevertheless, Borgani et al. 2004 have recently reported preheating simulations that can reproduce both observed $M-T$ and $L-T$ relations by adopting the same analysis procedure used for analyzing the observational data.)

In fact, the entropy profiles in galaxy groups derived from the ASCA data indicated that preheating likely occurred late in time, around $z=2-2.5$ (Finoguenov et al. 2002). Assuming outflow heating and using the constraint on the inverse Compton scattering of cosmic microwave background (CMB) photons by AGN hot spots, Yamada \& Fujita (2001) also reached a similar conclusion in favor of late preheating.

Radiative cooling that condenses the intracluster gas into stars has also been suggested to be a viable alternative for breaking the similarity scaling laws observed in the X-ray (e.g., Voit \& Bryan 2001; Wu \& Xue 2002). However, uncertainties pertinent to the delicate balance of cooling by some level of heating remain to be resolved so as to avoid catastrophic cooling (Tornatore et al. 2003).

Parallel to the developments in cluster physics, the Cosmic Background Imager (CBI) experiment recently discovered a higher level of temperature anisotropy than anticipated by $3 \sigma$, around the harmonic mode number $2000<l<3000$, in the CMB radiation (Pearson et al. 2003). This feature was attributed to the Sunyaev-Zel'dovich (SZ) effects (Bond et al. 2002). The Arcminute Cosmology Bolometer Array Receiver (ACBAR) experiment also detected high- $l$ excess even more recently (Kuo et al. 2004), which has also been attributed to the SZ signals (Goldstein et al. 2003).

To account for the high level of SZ signals, the value of $\sigma_{8}$ was estimated to be 1 by these collaborations, although a slightly larger $\sigma_{8}$ fits the CBI data better. The SZ power is known to depend sensitively on $\sigma_{8}$ (Komatsu \& Seljak 2002; Zhang et al. 2002), and the SZ effect has been thought to be a unique probe to narrow down $\sigma_{8}$ to high precision (Fan \& Chiueh 2001). In fact, the galaxy distribution combined with the distant supernova and $\mathrm{CMB}$ data has already constrained $\sigma_{8}$ within a window $0.75<\sigma_{8}<1$ (Bond et al. 2002). On the other hand, the cluster abundance study of the Sloan Digital Sky Survey finds that $\sigma_{8} \Omega_{m}^{0.6}=0.35 \pm 0.03$ (Bahcall et al. 


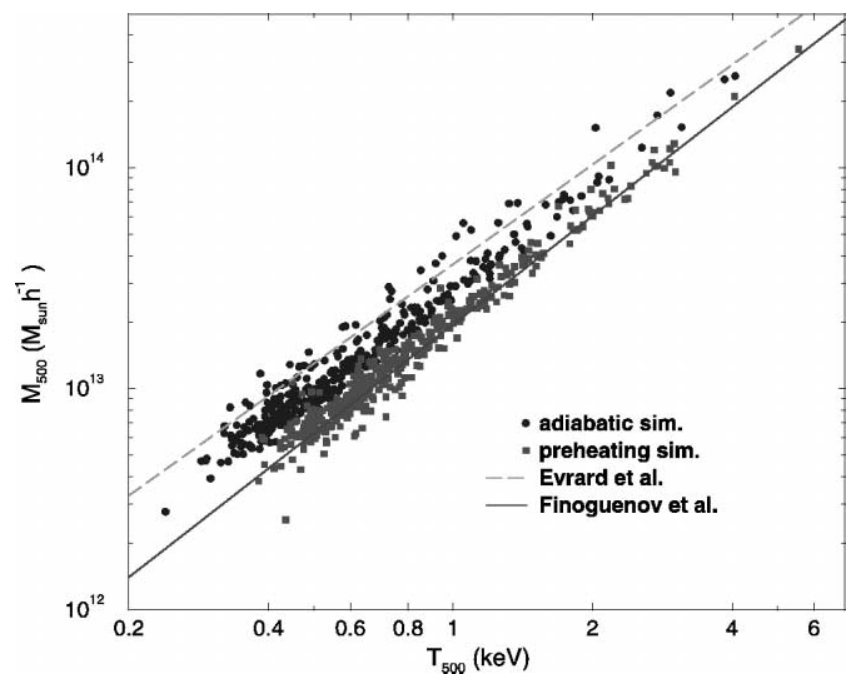

Fig. 1. $-M_{500}-T_{500}$ relation. Filled circles represent the adiabatic simulation data for $\sigma_{8}=0.94$, to be compared with the long-dashed line, the fitting formula of Evrard et al. (1996). Filled squares are the preheating simulation data for $\sigma_{8}=0.94$ and are consistent with the solid line, the observational data fit given in Finoguenov et al. (2001). [See the electronic edition of the Journal for a color version of this figure.]

2003), consistent with results of X-ray cluster abundance (Reiprich \& Böhringer 2002; Rosati et al. 2002 and references therein). The extreme value $\sigma_{8}=1$ thus demands a rather low matter-density parameter, $\Omega_{m}=0.175 \pm 0.025$.

If the SZ effect indeed accounts for the high- $l$ excess of CBI and ACBAR, preheating will exacerbate the awkward situation of the already large $\sigma_{8}$. Past works have indicated that preheating reduces the SZ flux (Bialek et al. 2001; da Silva et al. 2001; White et al. 2002). These works were, however, unable to recover both observed $M-T$ and $L-T$, rendering the reduction of SZ flux not amenable to calibration by X-ray observations.

Motivated by the evidences of late preheating around $z=$ 2 , we study a local heating scheme, which recovers both observed $M-T$ and $L-T$. This Letter is confined to addressing the relevance of such heating to the interpretation of CBI and ACBAR high- $l$ results. Details of other aspects will be reported elsewhere.

\section{SIMULATIONS AND $M-T$ AND $L-T$ RELATIONS}

We ran cosmological hydrodynamic simulations with the GADGET code (Springel et al. 2001b) for both $\sigma_{8}=0.94$ and 1 cases in a periodic box of comoving size $100 \mathrm{~h}^{-1} \mathrm{Mpc}$. The $\sigma_{8}=1$ run continues the $\sigma_{8}=0.94$ case beyond the present and is then scaled back to the present epoch with different cosmological parameters and a higher $\sigma_{8}$. The simulations contain $256^{3}$ dark matter particles and $256^{3}$ gas particles, with the gravitational softening length being $20 \mathrm{~h}^{-1} \mathrm{kpc}$ and the mass resolution $4.8 \times 10^{9} h^{-1} M_{\odot}$ for the dark matter and $0.83 \times$ $10^{9} h^{-1} M_{\odot}$ for the gas. All runs start at $z=100$ with the same initial conditions for $\left(n_{s}, \sigma_{8}, \Omega_{m}, \Omega_{b}, \Omega_{\Lambda}, h\right)=(1,0.94,0.34$, $0.05,0.66,0.66)$.

Heat injection occurs at $z=2$ and assumes a densitydependent form,

$$
\Delta u=u_{0} \exp \left(-\frac{\rho_{b 0}}{\rho_{b}}\right)
$$

where $\rho_{b}$ and $\rho_{b 0}$ are the local and background baryon densities, respectively, and $u$ is the specific internal energy. A single parameter $u_{0}$ is to be tuned to fit the observed $M-T$ and $L-T$ relations described below. This form of heating ensures that the highest density region receives a constant specific energy $u_{0}$ while the lowest density region receives no heat. The injected entropy has a local minimum at the core of a bound object and maximum at the outskirts around $\rho_{b} \sim \rho_{b 0}$. The entropy injection scheme of Borgani et al. (2001b, 2002) is similar, although not in detail, to our local heating scheme, but it failed in producing the observed $M-T$ relation.

We find the best value of $u_{0}$ to correspond to $7 \mathrm{keV}$ temperature, and the amount of injected heat is equivalent to giving every baryon $0.75 \mathrm{keV}$ temperature on average. Although the local injected heat is rather high, the averaged injected heat is comparable to, or even lower than, that suggested by recent studies (Loewenstein 2000; Wu et al. 2000; Bower et al. 2001; Borgani et al. 2001a, 2002; Xue \& Wu 2003). Since our heating occurs late in time and since a present-day cluster of $10 \mathrm{keV}$ is still deficient in X-ray luminosity (e.g., Fig. 2), we find this amount of local heat injection not unreasonable.

A standard friends-of-friends algorithm with a 0.2 linking length is employed to locate halos. The mass centers may be considerably displaced from the peaks of spherical overdensity, such as in the case of major mergers. In these cases, the overdensity so obtained is not useful for testing the $L-T$ and $M-T$ relations. They amount to about $20 \%-30 \%$ of all halos with X-ray bolometric luminosity greater than $10^{41} \mathrm{ergs} \mathrm{s}^{-1}$ and are avoided by selecting only those with nonzero $M_{2500}$, where $M_{n}$ represents the mass within a sphere of overdensity $n$ times that of the critical density.

The temperature $T$ of an ionized plasma is related to the internal energy per mass $u$ of the neutral fluid in hydrodynamic simulations by $k_{B} T=(2 / 3) \mu m_{p} u$, where $\mu$ is the mean "molecular" weight of $e^{-}, p^{+}$, and $\mathrm{He}^{2+}$ and is equal to 0.588 . In addition, we define the bremsstrahlung emission-weighted temperature as $\left\langle n_{e}^{2} T^{3 / 2}\right\rangle /\left\langle n_{e}^{2} T^{1 / 2}\right\rangle$, to be compared with the measured temperature of X-ray continuum.

Figure 1 compares both adiabatic and preheating $M_{500}-T_{500}$ relations from our $\sigma_{8}=0.94$ simulations with the fitting formula for adiabatic simulations given by Evrard et al. (1996) and with the fitting formula of HIFLUGCS data given by Finoguenov et al. (2001). Plotted here are only data with bolometric luminosities higher than $10^{41}$ ergs $\mathrm{s}^{-1}$. The $M_{500}-T_{500}$ relation of our adiabatic simulation, which has a slightly steeper slope, agrees fairly with that of Evrard et al. But both yield too low a temperature in bound objects. On the other hand, our preheating simulation yields an $M_{500}-T_{500}$ relation agreeing very well with the observations.

A constant metallicity, 0.3 times the solar value, is adopted here to compute the X-ray bolometric luminosity, which reads

$$
L_{500}=1.4 \times 10^{-27} \int T^{1 / 2} g(T) n_{e}\left(\sum_{i} n_{i} Z_{i}^{2}\right) d V
$$

in units of ergs $\mathrm{s}^{-1}$, where the volume integration is up to $R_{500}$, the summation is over different ion species, $Z$ is the atomic number, and $g(T)$ is the Gaunt factor (Mathiesen \& Evrard 2001). Figure 2 shows $L_{500}$ against $T_{500}$. Also shown are the observed $L_{500}$ and $T_{500}$ compiled by Arnaud \& Evrard (1999), Helsdon \& Ponman (2000), and Markevitch (1998). The preheating result for the $\sigma_{8}=0.94$ case again shows good agreement with X-ray observations, except for some excess luminosity at galaxy group scale with $T_{500}<0.6 \mathrm{keV}$. Mulchaey 


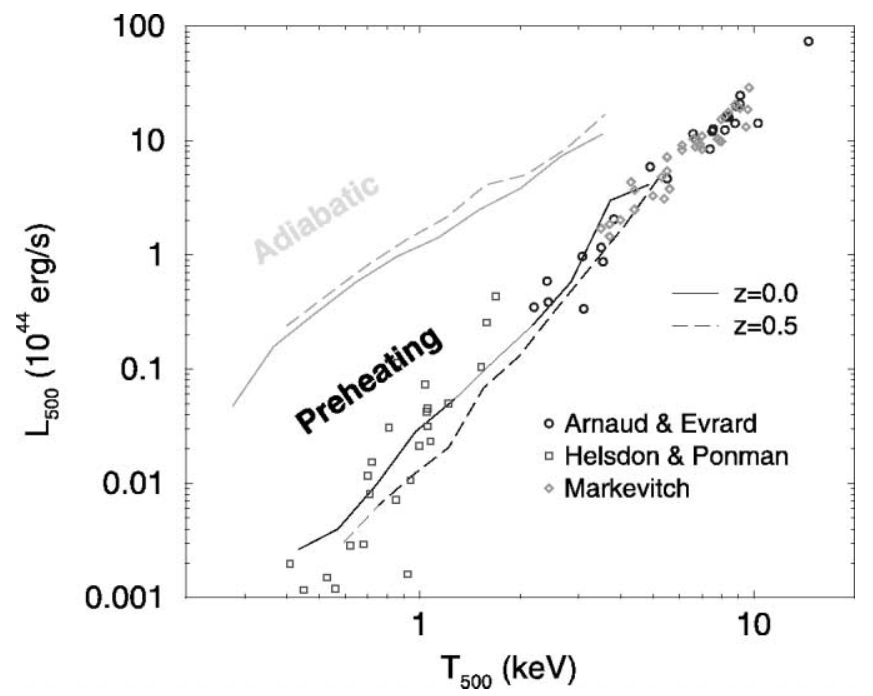

FIG. 2. $-L_{500}-T_{500}$ relation. Square, circle, and diamond symbols are the observed X-ray luminosities and temperatures. The solid and long-dashed lines are the simulation results for $\sigma_{8}=0.94$ at $z=0$ and 0.5 , respectively. The top band is for the adiabatic simulations, and the bottom band is for the preheating simulations. [See the electronic edition of the Journal for a color version of this figure.]

(2000) pointed out that low-temperature X-ray samples were often observed up to only $50 \%$ of the virial radius, thus resulting in an artificial break in the observed $L-T$ data shown in Figure 2. No obvious low-temperature break is found in our results.

On the luminosity evolution, Ettori et al. (2004) recently reported $L-T$ consistent with mild negative evolution and Novicki et al. (2002) found weak or no evolution, but Vikhlinin et al. (2002) to the contrary found positive evolution. Our heating results, which compare $L-T$ of $z=0$ and 0.5 , support mild negative evolution or no evolution.

\section{SZ AND CMB POWER SPECTRA}

The agreement of our $M_{500}-T_{500}$ and $L_{500}-T_{500}$ relations with observations assures the proposed heating scheme to be well constrained by observations. We thus proceed to construct $1 \operatorname{deg}^{2} \mathrm{SZ}$ maps up to $z=2.5$, following the method described in da Silva et al. (2000). The average power spectra of 40 such SZ maps for each adiabatic and preheating simulation of $\sigma_{8}=0.94$ and 1 cases are shown in Figure 3. The adiabatic results are consistent with earlier GADGET results (Springel et al. 2001a). Both the $\sigma_{8}=0.94$ and 1 runs show that preheating reduces the averaged SZ power spectra by a factor of 2-3 over the angular scales $l=2000-4000$.

We employ the scaling formula (Komatsu \& Seljak 2002) and rescale the SZ power spectra of both $\sigma_{8}=1$ and 0.94 cases in Figure 3 from our original $\Omega_{b} h^{2}=0.018$ and 0.0218 , respectively, to $\Omega_{b} h^{2}=0.022$ used in Bond et al. (2002) for detailed comparison. The CBI deep-field observations scanned a $9 \mathrm{deg}^{2}$ sky (Pearson et al. 2003). We hence compute the preheated SZ power spectrum averaged over nine $1 \mathrm{deg}^{2} \mathrm{SZ}$ maps and add it to the (noise-free) primary CMB spectrum of $\Omega_{b}=0.04, \Omega_{m}=0.3$, and $\Omega_{\Lambda}=0.7$ used in Bond et al. (2002) to obtain one realization. A total of 40 realizations are constructed for checking the SZ cosmic variance. Clear in Figure 3 is that the preheated SZ + CMB power spectrum of $\sigma_{8}=1$ lies well below the $2 \sigma$ error box of CBI data, but it is consistent with ACBAR data (Kuo et al. 2004).

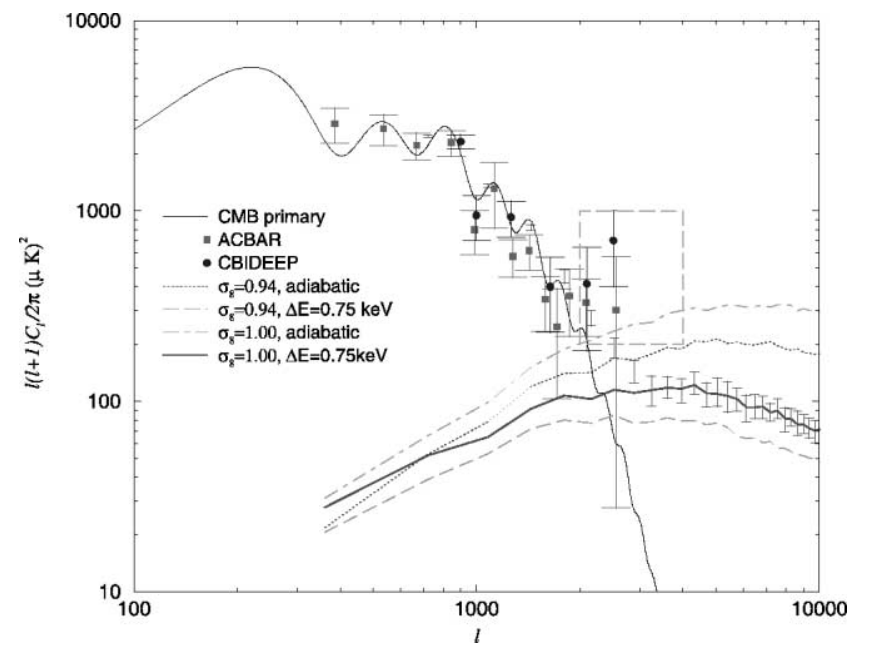

FIG. 3.-Comparison of CBI and ACBAR data with our preheating and adiabatic SZ power spectra of $\sigma_{8}=0.94$ and 1 and with our preheating $\mathrm{SZ}+\mathrm{CMB}$ spectrum of $\sigma_{8}=1$, where its $1 \sigma$ error bars arise from the cosmic variance of $9 \mathrm{deg}^{2}$. (The horizontal axis is the harmonic mode number $l$.) While the $\sigma_{8}=1$ adiabatic SZ spectrum comfortably intersects the CBI $2 \sigma$ error box over the band $l=2000-4000$, the preheating spectrum lies well below the CBI error box. [See the electronic edition of the Journal for a color version of this figure.]

\section{CONCLUSIONS}

In sum, the agreement of our preheating cosmological simulations with the observed $M_{500}-T_{500}$ and $L_{500}-T_{500}$ relations demonstrates that our impulsive local heating scheme can be a plausible prescription. Our quantitative prediction of reduced SZ flux is, in this regard, well constrained by the existing X-ray observations. The reduction of SZ flux must necessarily demand suitable modifications to the interpretation of the CBI and ACBAR deep-field results. Although the ACBAR high- $l$ excess is still consistent with the SZ effect of intracluster gas in a $\sigma_{8}=1$ concordance world model, the CBI high- $l$ excess can unlikely result entirely from the SZ effects of this world model.

Using the $\sigma_{8}^{7}$ scaling law of Komatsu \& Seljak (2002) to estimate the preheated SZ flux, we find that $\sigma_{8}$ must be raised to 1.2 for SZ effects to explain the CBI high- $l$ data. But $\Omega_{m}$ is then pushed down to $0.13 \pm 0.02$, constrained by $\sigma_{8} \Omega_{m}^{0.6}=$ $0.35 \pm 0.03$ (Bahcall et al. 2003). Such an $\Omega_{m}$ is too low for the big bang nuclear synthesis model to be consistent with the observed X-ray baryon fraction $\left(\leq_{4}^{1}\right)$ in massive clusters (Ettori \& Fabian 1999; Mohr et al. 1999). Various explanations, other than SZ effects, for the CBI high- $l$ excess have also been proposed, and they were discussed in Pearson et al. (2003).

We now comment on the Berkeley-Illinois-Maryland Association (BIMA) blank-field observation around $3000<l<$ 6000 , which was claimed to place an upper limit, $12.4 \mu \mathrm{K}$, on the SZ power with 95\% confidence (Dawson et al. 2002). Although our preheating results shown in Figure 3 satisfy this constraint, we find the BIMA survey field $\left(0.25 \mathrm{deg}^{2}\right)$ too small, and thus vulnerable to large sample variance, to place a viable constraint on the SZ power. Moreover, BIMA observations made attempts to avoid radio sources, which are likely correlated with galaxy clusters. Hence the BIMA data can be antibiased against SZ sources.

We finally remark that radiative cooling has been entirely ignored in the present work for the following reason. As of today, cosmological hydrodynamic simulations incorporating radiative cooling (e.g., Lewis et al. 2000; Pearce et al. 2000; Muanwong et al. 2001, 2002; da Silva et al. 2001; Tornatore 
et al. 2003) have either suffered from a cooling catastrophe in producing an excessively large amount of cooled condensates to be identified as star clusters or resorted to unphysical means to suppress the coolants. More fundamental to this problem is that certain low-level continual heating from star-forming activities, supernovae, and AGNs must necessarily proceed quiescently and delicately in preventing the runaway cooling. Any sophisticated modeling so far for these feedback activities has at best been ad hoc, and its reliability is difficult to assess in detail.

We thank M. Birkinshaw, C. J. Ma, U. Pen, K. Umetsu, J. H. P. Wu, X.-P. Wu, Y.-J. Xue, and P. Zhang for various helpful discussions. Support from NSC of Taiwan, under the grant NSC92-2112-M-002-021, and use of the NCHC computing facilities are acknowledged.

\section{REFERENCES}

Allen, S. W., \& Fabian, A. C. 1998, MNRAS, 297, L57

Allen, S. W., Schmidt, R. W., \& Fabian, A. C. 2001, MNRAS, 328, L37

Arnaud, M., \& Evrard, A. E. 1999, MNRAS, 305, 631

Bahcall, N. A., et al. 2003, ApJ, 585, 182

Bialek, J. J., Evrard, A. E., \& Mohr, J. J. 2001, ApJ, 555, 597

Bond, J. R., et al. 2002, preprint (astro-ph/0205386)

Borgani, S., et al. 2001a, ApJ, 559, L71

2001b, ApJ, 561, 13

2002, MNRAS, 336, 409

2004, MNRAS, 348, 1078

Bower, R. G., et al. 2001, MNRAS, 325, 497

Chiueh, T., \& He, X. G. 2002, Phys. Rev. D, 65, 123,518

da Silva, A. C., Barbosa, D., Liddle, A. R., \& Thomas, P. A. 2000, MNRAS, 317, 37

da Silva, A. C., et al. 2001, ApJ, 561, L15

David, L. P., et al. 2001, ApJ, 557, 546

Dawson, K. S., et al. 2002, ApJ, 581, 86

Ettori, S., \& Fabian, A. C. 1999, MNRAS, 305, 834

Ettori, S., et al. 2004, A\&A, 417, 13

Evrard, A. E., \& Henry, J. P. 1991, ApJ, 383, 95

Evrard, A. E., Metzler, C. A., \& Navarro, J. F. 1996, ApJ, 469, 494

Fan, Z., \& Chiueh, T. 2001, ApJ, 550, 547

Finoguenov, A., Jones, C., Böhringer, H., \& Ponman, T. J. 2002, ApJ, 578, 74

Finoguenov, A., Reiprich, T. H., \& Böhringer, H. 2001, A\&A, 368, 749

Goldstein, J. H., et al. 2003, ApJ, 599, 773

Helsdon, S. F., \& Ponman, T. J. 2000, MNRAS, 319, 933

Kaiser, N. 1986, MNRAS, 222, 323

Komatsu, E., \& Seljak, U. 2002, MNRAS, 336, 1256

Kuo, C. L., et al. 2004, ApJ, 600, 32

Lewis, G. F., et al. 2000, ApJ, 536, 623

Loewenstein, M. 2000, ApJ, 532, 17

Markevitch, M. 1998, ApJ, 504, 27
Mathiesen, B. F., \& Evrard, A. E. 2001, ApJ, 546, 100

Mohr, J. J., Mathiesen, B. F., \& Evrard, A. E. 1999, ApJ, 517, 627

Muanwong, O., Thomas, P. A., Kay, S. T., \& Pearce, F. R. 2002, MNRAS, 336, 527

Muanwong, O., et al. 2001, ApJ, 552, L27

Mulchaey, J. S. 2000, ARA\&A, 38, 289

Navarro, J., Frenk, C. S., \& White, S. D. M. 1996, ApJ, 462, 563

Novicki, M. C., Sornig, M., \& Henry, J. P. 2002, AJ, 124, 2413

Pearce, F. R., Thomas, P. A., Couchman, H. M. M., \& Edge, A. C. 2000, MNRAS, 317, 1029

Pearson, T. J., et al. 2003, ApJ, 591, 556

Peterson, J. R., Kahn, S. M., Paerels, F. B. S., Kaastra, J. S., Tamura, T., Bleeker, J. A. M., Ferrigno, C., \& Jernigan, J. G. 2003, ApJ, 590, 207

Ponman, T. J., Bourner, P. D., Ebeling, H., \& Böhringer, H. 1996, MNRAS, 283, 690

Reiprich, D. E., \& Böhringer, H. 2002, ApJ, 567, 716

Rosati, P., Borgani, S., \& Norman, C. 2002, ARA\&A, 40, 539

Shapiro, P. R., \& Iliev, I. T. 2002, ApJ, 565, L1

Springel, V., White, M., \& Hernquist, L. 2001a, ApJ, 549, 681

Springel, V., Yoshida, N., \& White, S. D. M. 2001b, NewA, 6, 79

Tabor, G., \& Binney, J. 1993, MNRAS, 263, 323

Tornatore, L., Borgani, S., Springel, V., Matteucci, F., Menci, N., \& Murante, G. 2003, MNRAS, 342, 1025

Vikhlinin, A., et al. 2002, ApJ, 578, L107

Voit, G. M., \& Bryan, G. L. 2001, Nature, 414, 425

White, M., Hernquist, L., \& Springel, V. 2002, ApJ, 579, 16

Wu, K. K. S., Fabian, A. C., \& Nulsen, P. E. J. 2000, MNRAS, 318, 889

Wu, X.-P., \& Xue, Y.-J. 2002, ApJ, 572, L19

Xue, Y.-J., \& Wu, X.-P. 2000, ApJ, 538, 65 2003, ApJ, 584, 34

Yamada, M., \& Fujita, Y. 2001, ApJ, 553, L145

Zhang, P. J., Pen, U.-L., \& Wang, B. 2002, ApJ, 577, 555 